\title{
Diversidad, pluralismos, reducciones en la biología: análisis de las relaciones entre nociones de gen*
}

Diversity, Pluralisms and Reductions in Biology: An Analysis of the Relationships between Gene Concepts

Guillermo Folguera ${ }^{\dagger}$

Nahuel Pallitto ${ }^{\ddagger}$

\begin{abstract}
Resumen
Actualmente, en la biología pueden encontrarse diferentes nociones de gen. Respecto a la relación entre ellas, Moss ha sostenido que en particular las nociones de gen clásico (gen-P) y gen molecular (gen-D) son independientes. En este trabajo se analiza la relación entre gen-P y gen-D en diferentes áreas de la biología tales como genética clásica, biología molecular, genética de poblaciones y neurobiología. Se propone aquí que dichas nociones no sólo presentan un fuerte vínculo conceptual, sino que en la bibliografía especializada se reconocen nociones "híbridas". Este doble escenario no sólo se distancia de posiciones de tipo pluralistas, sino que abona tesis reduccionistas al seno de la propia biología de la actualidad.
\end{abstract}

Palabras clave: gen-P - gen-D - reducción - monismo - pluralismo

\begin{abstract}
Currently, it is possible to find different notions of genes in Biology. Regarding their relationship, Moss has mentioned that the notions of classic gene (gene-P) and molecular gene (gene-D) are independent. In this work, we analyze the relationship between gene-P and gene-D in different areas of Biology such as Genetics, Molecular Biology, Genetics of Populations and Neurobiology. We propose that these notions not only present an important conceptual link but that different "hybrid" notions are also recognized in the specialized bibliography. We consider that, against any kind of pluralism, this dual conceptualization further supports reductionist thesis in contemporary Biology.
\end{abstract}

Keywords: gene-P - gene-D - reduction - monism - pluralism

\footnotetext{
* Recibido: 30 de Marzo de 2016. Aceptado con revisiones: 5 de Diciembre de 2016.

+ Grupo de Filosofía de la Biología, Universidad de Buenos Aires/CONICET. Para contactar a los autores, por favor, escribir a: guillefolguera@yahoo.com.ar.

* Grupo de Filosofía de la Biología, Universidad de Buenos Aires/CONICET.

Metatheoria 8(2)(2018): 63-73. ISSN 1853-2322.

(C) editorial de la Universidad Nacional de Tres de Febrero. Publicado en la República Argentina.
} 


\section{Introducción}

Desde su nacimiento, la biología ha mostrado una gran proliferación de áreas y campos del conocimiento, tendencia que se ha acelerado en las últimas décadas. En este sentido, puede reconocerse el origen de campos de conocimiento diversos tales como: neurobiología, biología molecular, fisiologías, ecología del comportamiento, ecología de poblaciones, ecología de comunidades, ecología de ecosistemas, genética de poblaciones, evo-devo, ecología de paisaje, macroecología, entre tantos otros. Como es de esperar, dentro de cada una de estas áreas se suelen utilizar términos específicos, los cuales están relacionados a su vez con otros conceptos, presentando en muchos casos una relación estrecha con la propia práctica científica. Sin embargo, en ocasiones la diferencia entre áreas se manifiesta también en cuanto al significado que posee un mismo término, mostrando así una polisemia significativa dentro de la propia biología. En este sentido, uno de los términos que ha sido indagado por numerosos autores por su notable polisemia es el de gen (para una buena aproximación ver, por ejemplo, Goldbach \& El-Hani 2008).

Dentro de esta diversidad, algunos conceptos parecieron ser más preponderantes y/o frecuentes que otros. Así, Waters (1994) señala la distinción entre el concepto clásico y el molecular. En sus palabras, mientras el concepto clásico de gen "involves its connection to the genotype/phenotype relation" (1994, p. 170), el concepto molecular "is that of a gene for a linear sequence in a product at some stage of genetic expression [...]. These products come at successive stages and include primary RNA transcripts, processed RNA, and polypeptides" (1994, p. 178). Otros autores, tales como Paul Griffiths y Karola Stotz (2006) reconocen al menos tres nociones de gen diferentes, una de las cuales surge luego de la emergencia de la postgenómica. De este modo, en la biología convivirían una noción instrumental de gen -aquella que los considera factores en modelos de transmisión fenotípica-, una noción nominal de gen -aquella que los concibe como secuencias delimitadas de ADN-y una noción postgenómica de gen -aquella que los concibe como templados en la producción de moléculas, no siempre bien delimitados, y los ubica en el marco de un sistema de desarrollo complejo-. Respecto a la última noción presentada, Stotz (2006) comenta: “'Genes' are not predetermined entities lined up in the genome like beads on a string; rather they are 'things an organism can do with its genome' on the spot to create a template resource for a product a cell may need at any particular time" (2006, p. 905). En un sentido similar a Waters -aun con diferencias-, Moss (2003) señala que "While defined by a predictable relationship to a phenotype, Gene-P, by contrast, is indeterminate with respect to DNA structure, that is, with regard to specific nucleic acid sequence" (2003, p. 41). A su vez, enfatiza que "But as we've already said, Gene-P is indeterminate with respect to its physical referent. It is indeterminate with respect to its DNA sequences and so there is not a certain something that is being transmitted, but rather an uncertain something" (2003, p. 42). El gen-D, por el contrario, se encuentra definido por una secuencia de ADN y sirve de templado para la producción de moléculas, siendo indeterminado con respecto al fenotipo. Dice Moss: "If some stretch of DNA provides the template out of which a strand of RNA of complementary sequence is produced, then for a biologist, working at the cell and molecular level, this counts as a gene" (2008, p. 42). ${ }^{1}$

Sin embargo, si bien los diferentes conceptos han sido analizados de manera aislada, poco se ha abordado el tipo de relación que poseen entre ellos. Por ejemplo, Moss señala que ambos conceptos no presentarían ninguna conexión, salvo en términos meramente retóricos - "rhetorical glue of the gene-as-

\footnotetext{
${ }^{1}$ Mientras Moss se centra en un par de conceptos, otros autores han marcado una diversidad mayor (Griffiths \& Neumann 1999, Grifftihs \& Stotz 2006, Stotz, Griffiths \& Knight 2004). Así, por ejemplo, Meyer, Bomfim y El-Hani (2013) señalan: "De hecho, nosotros pensamos que hay más conceptos relativos a los genes que son usados en la comunidad científica que gen-P y gen-D” (2013, p. 12). En particular, en este trabajo evitaremos dicho debate y nos focalizaremos en una de las clasificaciones que han tenido mayor impacto, que es la presentada por Moss en relación con los términos gen-P y gen-D. A su vez, consideramos que la clasificación de Moss muestra afinidades, a los fines del presente trabajo, con las nociones instrumental, nominal y postgenómica de gen presentada por Griffiths y Stotz (el gen instrumental sería análogo al gen-P y los genes nominal y postgenómico caen bajo la caracterización de gen-D). De este modo, consideramos innecesaria una distinción mayor como la propuesta por dichos autores.
} 
text metaphor" (Moss 2002, 2003)-. A partir de dicha no-conexión, Moss (2003) plantea que debemos aceptar un escenario de tipo diverso: "Far from attempting to absorb or assimilate one to the other, the real objective should be to disentangle the two, recognize their independent and nonoverlapping status, and thereby undercut misleading inferences derived from their unwarranted conflation" (2003, p. 58). Es preciso aquí contextualizar dicho comentario, en tanto que la "relación" entre ambos conceptos posición que Moss rechaza- sería en todo caso un tipo de reducción. Cabe recordar que se han registrado numerosos intentos de reducción de la genética clásica a la genética molecular y que en general no se han considerado exitosos a los mismos. De este modo, el razonamiento de Moss parece ser el siguiente: ante el fracaso de la reducción de la genética clásica a la genética molecular, y la presencia del mismo término con diferentes significados, es imperioso desistir en estrategias de fusión. A su vez, es importante reconocer que la "independencia" sostenida por Moss, parece derivar entre otros elementos en la ausencia de "híbridos", esto es, nociones de gen que presenten un carácter mixto entre clásico y molecular:
Would it not simply be a matter of empirically fleshing out what the structure of the physical referent would be? The answer is no. And the reason the answer is no is that what one almost always, if not always, finds in cases of Gene-P, is that it is not the presence of some specific sequence that correlates with the appearance of a phenotype, but rather the absence of some 'normal' sequence resource that is at issue, and there are always many ways for something to be absent. There are, for example, over 900 different documented DNA sequences that may show up as 'the gene for cystic fibrosis'. Likewise, the assurance that two blue-eyed parents may be given that a child of theirs would also have light-coloured eyes is based upon the assumption that neither blue-eyed parent has the genetic resource for producing brown-eye pigment, however it is that they may be lacking it. What is inherited, what is passed on, is the lack of something, the lack of a normal resource for producing brown eye pigment (Moss 2008, p. 42).

A partir de lo señalado hasta aquí, podemos resumir la propuesta de Moss en cuatro tesis respecto a la temática que nos interesa:

i. que respecto a la noción de gen no hay un único concepto utilizado al seno de la comunidad científica;

ii. que son dos los conceptos más utilizados en biología: gen-P y gen-D;

iii. que no es posible reconocer investigaciones que operen con conceptos "híbridos" entre el gen-P y el gen-D;

iv. que dichos conceptos son independientes entre sí.

De estas cuatro tesis, el autor desprende la necesidad de conservar este dualismo conceptual en la biología evitando estrategias deflacionarias como la reducción.

En este trabajo nos focalizaremos en indagar y problematizar el tipo de vínculo que presentan entre sí el gen-P y el gen-D. En particular, sostendremos un acuerdo general con la tesis i, no profundizaremos en la tesis ii (aunque sí utilizaremos el aparato conceptual presentado por Moss), pero a diferencia de Moss intentaremos sostener la idea que en las investigaciones actuales en biología se reconoce la presencia de híbridos (iii), a la vez que entre el gen-P y el gen-D no se reconoce tal independencia (iv). De lo anterior, se desprende una consecuencia muy diferente a la de Moss: que la persistencia del dualismo gen-P y gen-D lejos de tratarse de una estrategia que promueve cierto pluralismo, se presenta hoy en la biología como uno de los elementos fundamentales que sostiene un tipo de monismo, fomentado a partir de estrategias de determinación y simplificación epistémica que pueden ser enmarcadas en un tipo de reducción de tipo explicativa. ${ }^{2}$ En cuanto a la metodología general utilizada, la misma consistirá en el análisis de textos, principalmente provenientes de revistas más recientes, tanto del campo de la filosofía de la biología, como de diversas áreas de la biología, tales

\footnotetext{
2 Por limitaciones de espacio, en este artículo se acota el análisis a los vínculos que mantienen las conceptualizaciones con un tipo de reduccionismo explicativo. No serán abordados, por lo tanto, otros niveles importantes de análisis como el semántico, el pragmático, el metodológico y el ontológico.
} 
como genética clásica, genética molecular, genética de poblaciones, genética del comportamiento y neurobiología, todos campos en los que la noción de gen es usada de manera frecuente. Con este objetivo y metodología, la estructura argumental es la siguiente: en la siguiente sección, se analizarán ejemplos prácticos de la noción de gen-P y gen-D que se presentan en diferentes campos del saber para una mejor comprensión conceptual. En la tercera sección se ahondará en la presencia de híbridos de dichos conceptos tratando de reconocer algunas de sus características diferentes. En la cuarta sección se indagarán las relaciones conceptuales entre dichos conceptos tratando de mostrar que no son independientes. Finalmente, en la quinta y última sección, se presentarán algunas de las principales conclusiones, respecto al vínculo entre dicho dualismo conceptual y el reduccionismo epistemológico explicativo en la biología.

\section{En busca de los genes P y D}

Como primer paso en nuestro recorrido, resulta fundamental comprender un poco mejor algunas de las principales características de los genes $\mathrm{P}$ y $\mathrm{D}$ en el contexto particular de sus aplicaciones. Veamos entonces ejemplos de la literatura especializada de ambos tipos de genes.

\subsection{Análisis de casos con genes-P}

Resulta claro que en la bibliografía especializada se encuentran de manera abundante ejemplos que utilizan la noción del gen-P. Sin embargo, hay algunas áreas en donde sin dudas estos ejemplos se reconocen especialmente conspicuos, tal como en el caso del área de la genética del comportamiento. En este sentido, nos centraremos aquí en dos trabajos, ambos recientes: el primero, denominado "MetaAnalysis of the Heritability of Human Traits Based on Fifty Years of Twin Studies", de Polderman et al. (2015), mientras que el segundo es "A Twin Study of the Genetics of High Cognitive Ability Selected from 11,000 Twin Pairs in Six Studies from Four Countries", de Haworth et al. (2009). En un primer análisis, es posible reconocer que se buscan aquí correlaciones entre el aspecto genético y los caracteres fenotípicos en gemelos, una de las vías de investigación frecuentes en dicha área. A su vez, en cuanto a las entidades biológicas que son señaladas, en general se limitan a los genes y a los organismos. Un claro ejemplo de ello es el estadístico comúnmente utilizado denominado "heredabilidad", el cual describe la medida en que las diferencias individuales en un rasgo fenotípico pueden ser atribuidas a diferencias genéticas. Los dos trabajos citados hacen uso de la misma herramienta metodológica y conceptual.

Ahora bien, identificadas las entidades, el siguiente interrogante es: ¿qué tipo de relación se reconoce entre las dos entidades exhibidas? Para ello, recordemos pues que, los modelos de variación genética desarrollados entre la década de 1950 y 1980, señalaban que la variación fenotípica tenía tres componentes: el ambiental, el genético y su interacción. Sin embargo, más allá del reconocimiento del ambiente como factor y de su interacción con la dimensión genética, puede verse que en gran parte de los trabajos estos dos factores pretenden ser minimizados, en oposición al aporte genético en la aparición de determinado fenotipo. Por ejemplo, en el caso del trabajo de Polderman et al. (2015) se explicita este privilegio del aporte genético y, en este mismo sentido un tipo de linealidad asumida en el vínculo entre las entidades señaladas. La contribución del ambiente, si bien mencionada, suele ser vista apenas como desvío de un valor esperado, una idea de ruido, despreciando cualquier valor epistémico del mismo. ${ }^{3}$ Por otro lado, en cuanto a las interacciones, se sabe que la metodología que incluye gemelos no permite estimar de manera adecuada sus efectos (Haworth et al. 2009).

A partir de lo señalado, es claro que el gen clásico encuentra, tal como sugería Moss, su determinación en el propio fenotipo, a la vez que se reconoce una clara omisión de las entidades correspondientes a los diferentes niveles de organización comprendidos entre el genético y el

\footnotetext{
${ }^{3}$ En este sentido se señaló previamente que el ambiente y la interacción suelen ser minimizados. Uno de los objetivos principales de la genética del comportamiento consiste en la identificación de genes particulares y el estudio de sus efectos (Plomin et al 2013), siendo los dos factores antes mencionados obstáculos epistémicos que conviene, cuando no excluir, minimizar en los análisis.
} 
organísmico. En paralelo a dicha omisión, se asume un tipo de relación lineal entre ambas entidades, que conceptualiza al ambiente y a la interacción de la variación fenotípica con la genética de manera tangencial y/o necesarias de ser desatendidas (Figura 1A).

Figura 1

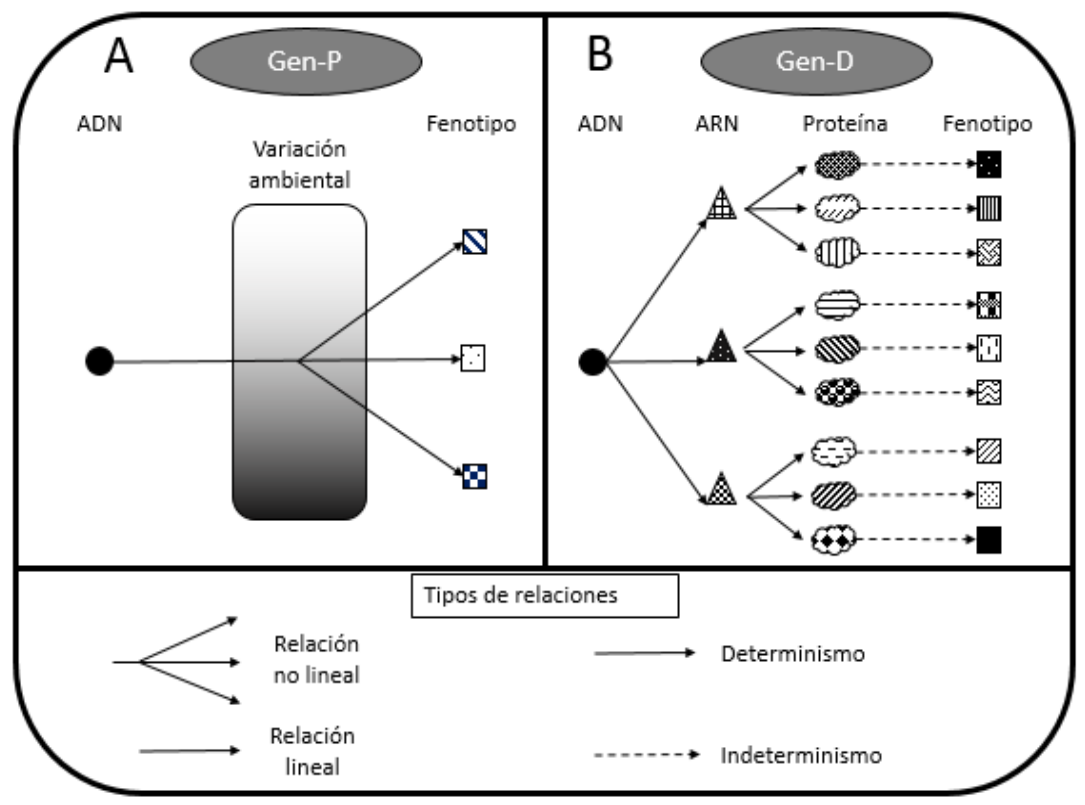

Diferentes modelos de Gen-P y Gen-D siguiendo la propuesta de Moss y algunas de las problematizaciones que se han dado al seno de la biología. A. El concepto de Gen-P es determinado con respecto al fenotipo, al margen de una relación compleja que involucra al ambiente y a la interacción, en muchos casos excluidos o minimizados. B. El concepto de Gen-D es determinado con respecto a ciertos productos moleculares pero indeterminado respecto al fenotipo.

\subsection{Análisis de casos con genes-D}

En lo que respecta a la presencia de los denominados genes-D en la bibliografía especializada, evidentemente éstos prevalecen en aquellos campos propios de la biología molecular y/o "aledaños". En efecto, no se trata de un uso exclusivo en los trabajos de dicha área, sino que ha permeado sobre otros campos tales como la biología evolutiva, biología del desarrollo, ecologías, entre otras. En particular, aquí nos hemos focalizado (sólo a modo de estudios particulares) en diversos estudios recientes del área de la biología molecular, tales como Celotto y Graveley (2001), Hickman y Cairns (2003), Scott et al. (1989) y Xiao-Min Li et al. (2015).

En principio, podemos reconocer que en estos trabajos la noción de gen aparece íntimamente vinculada a la de proteína y de ARN. A su vez, cabe señalar que en todos los casos, los fenotipos han aparecido como indeterminados, no reconociéndose el nivel de organismo. Respecto al tipo de relación que se presenta entre ADN-ARN y proteínas, cabe recordar que posteriormente al desarrollo del denominado dogma central de la biología molecular, la relación entre dichos conceptos ha sufrido numerosas complejizaciones. Así, por ejemplo, pueden reconocerse muchas problematizaciones en relación con dicho vínculo. Por ejemplo, el "encendido" y "apagado" de genes, el denominado "splicing alternativo", la jerarquización de los genes, así como la aparición en las últimas décadas en el escenario de las modificaciones epigenéticas, las cuales se proponen como unidades de herencia, junto a las genéticas. Del breve análisis realizado puede reconocerse no sólo un predominio de un tipo de noción de gen-D, sino que, en cuanto a su relación con los conceptos, éstos presentan una relación que puede entenderse de tipo no lineal (Hang 2015, Figura 1B). 
Ahora bien, hasta aquí hemos podido reconocer algunas de las principales diferencias entre las nociones $\mathrm{P}$ y $\mathrm{D}$ en algunos de sus contextos de uso. Recordemos que en lo que hemos planteado como la tesis iv de Moss (véase Introducción), se sostiene una independencia entre ambos tipos de conceptos. Sin embargo, consideramos que de la mera diferencia no es posible derivar que los mismos sean efectivamente independientes. Sin embargo, esto resulta necesario de ser profundizado, aun cuando se acepte el fracaso efectivo de la reducción de la genética clásica a la genética molecular. Ciertamente, la independencia entre ambas nociones de gen recibe al menos dos grupos de cuestionamientos en los que resulta importante detenerse. Por un lado, sostendremos que se reconoce actualmente en investigaciones académicas algún tipo de noción "híbrida" de gen (sección III). Por otro lado, más allá de la aparición y consolidación, de hecho, de este tipo de noción, ambos grupos de genes presentan vínculos conceptuales necesarios de ser elucidados con importantes nexos con las reducciones de tipo explicativa en la biología (sección IV). A partir de lo señalado, es fundamental reconocer ambas situaciones, esto es, tanto en lo que refiere a sus investigaciones efectivas como en lo que refiere a las tradiciones que se encuentran estructurando sus programas de investigación.

\section{En busca de los híbridos P-D}

Como hemos visto en la primera sección, el análisis realizado por Moss descarta la posibilidad de trabajos de investigación que utilicen conceptos híbridos. Sin embargo, cierto es que en la bibliografía más reciente es posible encontrar diferentes trabajos en los que se reconocen tanto el uso del denominado gen-P como del gen-D. Sin ninguna intención de exhaustividad, sino sólo avanzar con el análisis señalado, veamos a continuación algunos de los trabajos que parecen presentar un carácter híbrido P-D.

El primer grupo de trabajos analizado refiere a la presencia de híbridos en la genética de poblaciones. Por ejemplo, en el trabajo de Nachman, Hoekstra y D'Agostino (2003) en el que aborda el caso del melanismo en el denominado ratón pocket, iqué noción de gen parece estarse utilizando? Es muy interesante, pues si tomamos en principio cómo está conceptualizado el gen, es claro que el fenotipo cumple el rol determinante, en este caso la variación del color de la población de ratones. Esto evidentemente lo encuadra en una de las características centrales del gen-P. ${ }^{4}$ Sin embargo, vemos que estrictamente no es un caso similar al tipo de gen-P antes presentado, en la medida que busca caracterizar las proteínas que median, aproximándose así a un tipo de gen productor de moléculas. ${ }^{5}$ Un análisis similar puede hacerse en otro trabajo de genética más del tipo funcional y menos evolutivo, tal como el de Sakudoh et al. (2007).

Otro campo en el que pueden reconocerse la presencia de híbridos de gen-P y gen-D es en la neurobiología. A fines de ciertos cuidados metodológicos en esta indagación nos centraremos en el análisis de aquellos que estudiaban la problemática del ADD/ADHD. Aquí fueron analizados numerosos trabajos entre los que se destacan, entre otros, los trabajos de Bellgrove et al. (2005), Heiser et al. (2004) y Swanson et al. (2000b).

En cuanto a la metodología utilizada, los niños son clasificados cualitativamente, discriminando aquellos que estarían afectados por el ADHD de aquellos no afectados. El grupo de niños no afectados pasa a cumplir el rol de "control" (Swanson et al. 2000a, p. 4755), en este sentido puede reconocerse que, al menos en una primera instancia, el determinante epistémico y metodológico es el fenotipo mismo. En términos fisiológicos, la teoría que goza de mayor aceptación en la bibliografía consultada es la teoría de dopamina del ADHD (Levy 1991, Swanson et al., 2007, Gonon 2008, Bellgrove et al. 2005, McGough 2005 y Faraone et al. 2005). En general se ha propuesto la presencia de una base genética (p.

\footnotetext{
4 Cabe recordar que algunos autores han presentado la acepción evolutiva de gen. En esta dirección, Williams define el gen como "eso que se segrega y recombina con frecuencia apreciable" (Williams 1966, p. 24).

5 Resulta pertinente hacer aquí una aclaración. Las mutaciones que se reconocen no siempre refieren a las secuencias codificantes, llamadas "genes estructurales". En ocasiones, las mutaciones se encuentran en zonas reguladoras. De todos modos, lo que se supone en todo caso es que las variantes modifican el tipo o la cantidad de una proteína determinada.
} 
ej., Pauls 2005, Vendruscolo et al. 2006, Smoot, Boothby \& Gillett 2007) y posteriormente se lo "verifica". En este sentido, es introducida la dinámica proteica, con lo que el gen presenta una nueva relación y, con ello, una resignificación. Los conceptos asociados son las moléculas, los órganos, el individuo, asumiendo una fuerte linealidad en las relaciones involucradas.

Pero volviendo al eje principal de nuestro trabajo, la presencia de conceptos híbridos en diferentes campos de la biología retrotrae la cuestión desarrollada por Moss, en relación con qué tipo de vínculo si acaso lo hay- entre ambos conceptos. Uno de los modos en que se puede avanzar con esta pregunta, es acerca de cuáles "pasos" son necesarios para pasar de una a otra conceptualización. ${ }^{6}$ Esto es, justamente, el tipo de análisis desarrollado a continuación.

\section{Las relaciones conceptuales entre el gen-P y el gen-D y algunas de sus consecuencias epistemológicas}

En esta instancia, a los fines de un análisis de la propuesta por Moss (tesis iv de la Introducción), cabe indagar cuáles son algunos de los presupuestos involucrados. Para ello, analizaremos las siguientes tres preguntas: ¿cómo conducirnos de lo reconocido como gen D (Figura 1B) al gen P (Figura 1A) o viceversa? ¿Qué tipos de relaciones hay entre ambas? Y finalmente, ¿qué tipo de relaciones presenta con escenarios de tipo reduccionista en la biología?

Partiremos en nuestra explicación del concepto de gen-D. Recordemos que dicho concepto era determinado con respecto a una secuencia de ADN pero indeterminado en cuanto al fenotipo. A su vez, se reconocía que las entidades moleculares están mediadas por relaciones complejas. El primer paso lógico que ocurre en el camino hacia el gen-P es la determinación del fenotipo por las proteínas (Figura 2A). Proteínas distintas causan fenotipos distintos. El segundo paso, consiste en la simplificación de las relaciones complejas entre las entidades moleculares (Figura 2B). De este modo, se transforman relaciones que no eran lineales (tal como el caso ADN-ARN-proteínas al seno de los propios estudios moleculares) en lineales. Nótese que los ejemplos brindados de genes híbridos se corresponden muy bien con este "momento" de la deconstrucción. Finalmente, a la simplificación realizada en el paso previo se le suma un "olvido" u omisión de las entidades intermedias entre el ADN y el fenotipo, dejándonos un esquema en el que los genes determinan el fenotipo, es decir, con el concepto de gen-P (Figura 2C).

\footnotetext{
${ }^{6}$ Estos "pasos" refieren a una forma lógica de concebir el vínculo y no a una secuencia metodológica que es necesario seguir de acuerdo al orden establecido.
} 
Figura 2



Reconocimiento y presentación de las diferentes "etapas" de la relación entre Genes-D y Genes-P. A. Se parte del gen-D. El primer paso consiste en el cambio en la relación propuesta entre proteína-fenotipo. Cada fenotipo es determinado por una proteína en particular. B. El segundo paso consiste en el cambio en la relación propuesta entre ADN-ARN-proteína. Las relaciones no lineales se conciben de forma lineal, obteniendo un tipo de concepto de gen-P-D. C. El tercer paso consiste en la omisión de las entidades ARN-proteína. Las entidades intermediaras entre el ADN y el fenotipo son excluidas, obteniendo el concepto de gen-P.

Del análisis desarrollado se desprende que:

(a) Hay un grupo de relaciones que conectan entidades de diferentes niveles de organización. En este sentido, puede reconocerse el tipo de relación reductiva que el propio Moss señala y que aún está fuertemente vigente dentro de los programas de investigación en la biología. De este modo, el dominio del fenotipo es "explicado" a partir del propio dominio molecular.

(b) La relación reductiva entre el dominio fenotípico y el molecular no es el único tipo de relación presente. También se presenta, hacia el seno del propio dominio molecular una relación de fuerte simplificación, que conlleva a transformar relaciones que no son lineales (tal como el caso ADN-ARN-proteínas al seno de los propios estudios moleculares) en lineales.

Ahora bien, ¿qué tipo de relaciones presenta con los reduccionismos epistemológicos de la biología? En el escenario que hemos visto, el vínculo entre los dos conceptos se establece a partir de procesos de determinación y de simplificación teórica. En la medida en que ambos conceptos "conviven" actualmente en la biología, se conforma un tipo de conceptualización común que admite versiones 
"intercambiables" al seno de la propia biología. En términos epistemológicos, el escenario es compatible con una interpretación reduccionista explicativa, en el cual las variaciones fenotípicas son explicadas mediante el ámbito genético, pero con el agregado de los procesos internos celulares con conectan al ADN (de manera simplificante) con otras entidades como las moléculas de ARN y las propias proteínas.

\section{Cierre y apertura: la biología diversa, la biología reductora, la biología plural}

Considerados en su conjunto, la presencia de ambos conceptos de genes -aún siendo marcadamente diferentes (sección II de este trabajo), la presencia de trabajos que presentan nociones híbridas (sección III) y el análisis teórico de cuáles son los supuestos que conllevan de un concepto a otro (IV)- sugieren que no sólo no observamos una independencia entre ambas nociones, sino que los reconocemos como parte de una misma estrategia reductiva aún presente en la biología.

A partir de lo señalado, se desprende que lejos de reconocer en la presencia de estos conceptos diversos una señal del pluralismo, más bien lo consideramos parte de una estrategia vigente dentro de los estudios de la biología contemporánea en clave monista. No se trata realmente de una diversidad plural de términos y conceptos en la biología actual. Lo que se trata, por el contrario, es de un programa de investigación que considera exitosas y necesarias las reducciones (de diferente naturaleza), identificaciones y simplificaciones en el estudio de los seres vivos. En este recorrido, hemos podido reconocer que la propia relación que se establece entre ambas nociones, es similar a las propias consideraciones que llevan a cabo los trabajos que consideran un término híbrido P-D.

Por último, es fundamental reconocer que lo señalado se trata de un programa de investigación, ya en acción en las ciencias de la vida y con gran relevancia. La filosofía de la biología debe tener como uno de sus objetivos el comprender el estado actual de la disciplina, tanto en la relación entre los aspectos epistémicos más generales y las prácticas científicas, como así también sus complejas relaciones internas. La diversidad conceptual al seno de la biología no necesariamente es un indicador de verdadera pluralidad. Las posiciones pluralistas deben sostener algún tipo de autonomía en cada uno de los ámbitos que la conforman. De esto no se deriva la ausencia de relaciones entre campos, pero sí que dichas relaciones deben tener un tipo simétrico y no uno marcadamente asimétrico, tal como en las propuestas reduccionistas (en cualquiera de sus formas), y que el modo de relacionarse no parta de un presupuesto de tipo lineal y simplificador. $\mathrm{O}$, dicho de otro modo, la mera diversidad terminológica y conceptual puede no ser expresión de una pluralidad, con campos autónomos y relaciones de tipo simétrica.

Bibliografía

Bellgrove, M.A., Hawi, Z., Lowe, N., Kirley, A., Robertson, I.H. y M. Gill (2005), "DRD4 Gene Variants and Sustained Attention in Attention Deficit Hyperactivity Disorder (ADHD): Effects of Associated Alleles at the VNTR and 521 SNP”, American Journal of Medical Genetics Part B (Neuropsychiatric Genetics) 136B: 81-86.

Celotto, A.M. y B.R. Graveley (2001), "Alternative Splicing of the Drosophila Dscam Pre-mRNA Is Both Temporally and Spatially Regulated”, Genetics 159: 599-608.

Faraone, S.V., Perlis, R.H., Doyle, A.E., Smoller, J.W., Goralnick, J.J., Holmgren, M.A. y P. Sklar (2005), "Molecular Genetics of Attention-Deficit/Hyperactivity Disorder”, Biological Psychiatry 57: 1313-1323.

Goldbach, T. y C.N. El-Hani (2008), "Entre receitas, programas e códigos: metáforas e ideias sobre genes na divulgação científica e no contexto escolar", Alexandria. Revista de educação em Ciência e Tecnologia 1: 153-189.

Gonon, F. (2008), "The Dopaminergic Hypothesis of Attention-Deficit Hyperactivity Disorder Needs Re-Examining", Trends in Neuroscience 32: 2-8. 
Griffiths, P.E. y E.M. Neumann-Held (1999), “The Many Faces of the Gene”, BioScience 49(8): 656-662.

Griffiths, P.E. y K. Stotz (2006), "Genes in the Postgenomic Era”, Theoretical Medicine and Bioethics 27: 499-521.

Hang, S. (2015), "Limits to Deterministic-Linear Causality in Biomedicine: Effects of Stochasticity and Non-Linearity in Molecular Network", en Bertolaso, M. (ed.), The Future of Scientific Practice: 'Bio-Techno-Logos', London: Pickering \& Chatto, pp. 41-64.

Haworth, C.M., Wright, M.J., Martin, N.W., Martin, N.G., Boomsma, D.I., Bartels, M., Posthuma, D., Davis, O.S., Brant, A.M., Corley, R.P., Hewitt, J.K., Iacono, W.G., McGue, M., Thompson, L.A., Hart, S.A., Petrill, S.A., Lubinski, D. y R. Plomin (2009), "A Twin Study of the Genetics of High Cognitive Ability Selected from 11,000 Twin Pairs in Six Studies from Four Countries", Behavior Genetics 39: 359-370.

Heiser, P., Friedel, S., Dempfle, A., Konrad, K., Smidt, J., Grabarkiewicz, J., Herpertz-Dahlmann, B., Remschmidt, H. y J. Hebebrand (2004), "Molecular Genetic Aspects of Attention-Defficit/Hyperactivity Disorder", Neuroscience and Biobehavioral Reviews 28: 625-641.

Hickman, M. y J. Cairns (2003), “The Centenary of the One-Gene One-Enzyme Hypothesis”, Genetics 163: 839-841.

Levy, F. (1991), "The Dopamine Theory of Attention Deficit Hyperactivity Disorder (ADHD)", Australian and New Zealand Journal of Psychiatry 25: 277-283.

Li, X.-M., Song, Y.-N., Xiao, G.-B., Zhu, B.H., Xu, G.C., Sun, M.Y., Xiao, J., Mahboob, S., Al-Ghanim, K.A., Sun, X.W. y J.T. Li (2015), "Gene Expression Variations of Red-White Skin Coloration in Common Carp (Cyprinus carpio)", International Journal of Molecular Sciences 16: 21310-21329.

McGough, J.J. (2005), "Attention-Deficit/Hyperactivity Disorder Pharmacogenomics", Biological Psychiatry 57: $1367-$ 1373.

Meyer, L.M.N., Bomfim, G.C. y C.N. El-Hani (2013), "How to Understand the Gene in the 21st Century”, Science $\mathcal{G}$ Education 22: 345-374.

Moss, L. (2002), What Genes Can't Do, Cambridge: MIT Press.

Moss, L. (2003), “One, Two (too?), Many Genes?”, The Quarterly Review of Biology 78: 57-67.

Moss, L. (2008), "The Meanings of the Gene and the Future of the Phenotype The Meanings of the Gene and the Future of the Phenotype", Genomics, Society and Policy 4: 38-57.

Nachman, M.W., Hoekstra, H.E. y S.L. D’Agostino (2003), "The Genetic Basis of Adaptive Melanism in Pocket Mice", Proceedings of the National Academy of Sciences 100: 5268-5273.

Pauls, D.L. (2005), "The Genetics of Attention-Deficit/Hyperactivity Disorder”, Biological Psychiatry 57: 275-282.

Plomin, R., DeFries, J., Knopik, V. y J. Neiderhise (2013), Behavioral Genetics, New York: Worth Publishers.

Polderman, T.J.C., Benyamin, B., de Leeuw, C.A., Sullivan, P.F., van Bochoven, A., Visscher, P.M. y D. Posthuma (2015), "Meta-Analysis of the Heritability of Human Traits Based on Fifty Years of Twin Studies", Nature Genetics 47: 702-709.

Sakudoh, T., Sezutsu, H., Nakashima, T., Kobayashi, I., Fujimoto, H., Uchino, K., Banno, Y., Iwano, H., Maekawa, H., Tamura, T., Kataoka, H. y K. Tsuchida (2007), "Carotenoid silk coloration is controlled by a CarotenoidBinding Protein, a Product of the Yellow Blood Gene”, Proceedings of the National Academy of Sciences 104: 89418946.

Scott, J., Wallis, S.C., Davies, M.S., Wynne, J.K., Powell, L.M. y D.M. Driscoll (1989), "RNA editing: A Novel Mechanism for Regulating Lipid Transport from the Intestine”, Gut Festschrift 30: 35-43.

Smoot, L.C., Boothby, L.A. y R.C. Gillett (2007), "Clinical Assessment and Treatment of ADHD in Children", International Journal of Clinical Practice 61: 1730-1738.

Stotz, K. (2006), "With 'Genes' Like That, Who Needs an Environment? Postgenomics's Argument for the 'Ontogeny of Information'”, Philosophy of Science 73: 905-917.

Stotz, K.; Griffiths, P.E. y R. Knight (2004), "How Biologists Conceptualize Genes: An Empirical Study”, Studies in History and Philosophy of Biological and Biomedical Sciences 35: 647-673.

Swanson, J., Oosterlaan, J., Murias, M., Schuck, S., Flodman, P., Spence, M.A., Wasdell, M., Ding, Y., Chi, H.C., Smith, M., Mann, M., Carlson, C., Kennedy, J.L., Sergeant, J.A., Leung, P., Zhang, Y.P., Sadeh, A., Chen, 
C., Whalen, C.K., Babb, K.A., Moyzis, R. y M.I. Posner (2000a), “Attention Deficit/Hyperactivity Disorder Children with a 7-Repeat Allele of the Dopamine Receptor D4 Gene Have Extreme Behavior but Normal Performance on Critical Neuropsychological Tests of Attention”, Proceedings of the National Academy of Sciences 97: 4754-4759.

Swanson, J.M., Flodman, P., Kennedy, J., Spence, M.A., Moyzis, R., Schuck, S., Murias, M., Moriarity, J., Barr, C., Smith, M. y M. Posner (2000b), "Dopamine Genes and ADHD”, Neuroscience and Biobehavioral Reviews 24: 21 25.

Swanson, J.M, Kinsbourne, M., Nigg, J., Lanphear, B., Stefanatos, G.A., Volkow, N., Taylor, E., Casey, B.J., Castellanos, F.X. y P.D. Wadhwa (2007), "Etiologic Subtypes of Attention-Deficit/Hyperactivity Disorder: Brain Imaging, Molecular Genetic and Environmental Factors and the Dopamine Hypothesis", Neuropsychology Review 17: 39-59.

Vendruscolo, L.F., Terenina-Rigaldie, E., Raba, F., Ramos, A., Naoto Takahashi, R. y P. Mormède (2006), "A QTL on Rat Chromosome 7 Modulates Prepulse Inhibition, a Neuro-Behavioral Trait of ADHD, in a Lewis $\mathrm{x}$ SHR intercross", Behavioral and Brain Functions 2: 21-27.

Waters, C.K. (1994), “Genes Made Molecular”, Philosophy of Science 61: 163-185.

Williams, G.C. (1966), Adaptation and Natural Selection, Princeton, NJ: Princeton University Press. 